\title{
Adherence to Asacol once daily versus divided regimen for maintenance therapy in ulcerative colitis: a prospective, multicenter, randomized study
}

\author{
Soo-Kyung Park ${ }^{1 *}$, Sang Hyun Park ${ }^{2 *}$, Chang Soo Eun ${ }^{3}$, Geom Seog Seo ${ }^{4}$, Jong Pil Im ${ }^{5}$, Tae Oh Kim ${ }^{6}$, Dong-Il Park ${ }^{1}$ \\ ${ }^{1}$ Division of Gastroenterology, ${ }^{2}$ Department of Internal Medicine, Kangbuk Samsung Hospital, Sungkyunkwan University School of Medicine, \\ Seoul; ${ }^{3}$ Department of Internal Medicine, Hanyang University Guri Hospital, Guri; ${ }^{4}$ Department of Internal Medicine, Digestive Disease \\ Research Institute, Wonkwang University Hospital, Wonkwang University College of Medicine, Iksan; ${ }^{5}$ Department of Internal Medicine and \\ Liver Research Institute, Seoul National University Hospital, Seoul National University College of Medicine, Seoul; ${ }^{6}$ Division of Gastroenterology, \\ Department of Internal Medicine, Haeundae Paik Hospital, Inje University College of Medicine, Busan, Korea
}

Background/Aims: A once-daily (OD) regimen of 5-aminosalicylic acid (5-ASA) was easier to comply with than a divided daily (DD) regimen, and that treatment efficacy for ulcerative colitis (UC) was not affected by the dosing regimen. This study evaluated treatment adherence of OD and DD dosing in the Korean UC patients. Methods: This study was a prospective, multicenter, randomized trial. UC patients were enrolled who have been in remission for more than 3 months. Patients were randomly assigned to the OD or DD group in a 1:1 ratio. The primary endpoint was adherence rate measured by tablet counts and selfreported adherence rate at 3, 6, 9, and 12 months. The relapse rate was measured at 1 year. Results: Data from the 180 patients who were randomized were analyzed. Both self-reported adherence rate and adherence rate measured by tablet counts were not different at every points, including 1 year. The patients' satisfaction of the OD group was higher than that of the DD group $(P<0.001)$. At 1 year, $91.2 \%$ and $95.5 \%$ of patients in the OD group and DD group had maintained clinical remission, respectively $(P=0.37)$. Conclusions: The adherence rates were not different between the OD group and DD group. The patients' satisfaction was higher in the OD group than in the DD group. 5-ASA OD dosing might have the same effect as DD for the maintenance of UC remission. (Intest Res 2019;17:349-356)

Key Words: Colitis, ulcerative; Mesalamine; Inflammatory bowel disease

\section{INTRODUCTION}

Treatments for UC aim to achieve remission by reducing inflammation of the mucosa and improving symptoms, and to

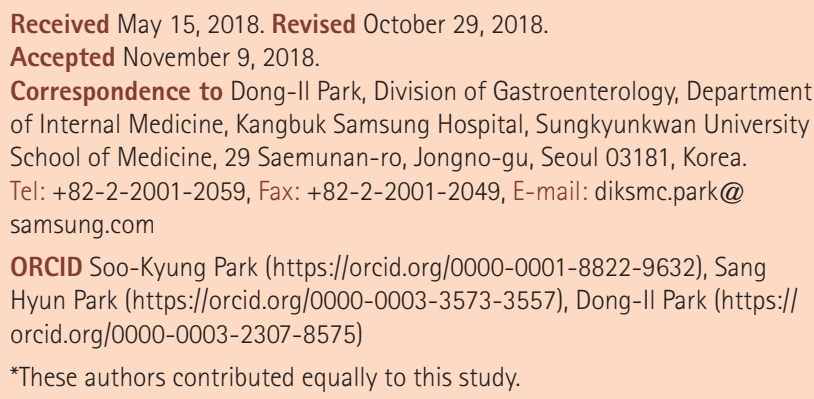

maintain remission to achieve good quality of life. Important factors affecting the choice of treatment plan include the severity, extent, and clinical features of the disease. 5-aminosalicylic acid (5-ASA) is currently established as a standard treatment for first-line treatment of mild-to-moderate UC. 5-ASA proved effective and is tolerable by the patients for the treatment of active disease and the maintenance of remission. ${ }^{1-3}$ Among 5-ASAs, mesalazine $\left(\right.$ Asacol $\left.^{\circledR}\right)$ has been widely used since the 1980s and is an effective medicine for the maintenance of UC remission..$^{4-9}$ Mesalazine is an aminosalicylate preparation that releases 5-ASA above $\mathrm{pH} 7$ and acts mainly in the terminal ileum and the right colon. ${ }^{10}$ Generally, a regimen of 1.6 to $2.4 \mathrm{~g}$ mesalazine divided into 2 or 3 daily doses is 
prescribed, and most Asacol ${ }^{\circledR}$ trials used thrice daily dosing schedules.

Continuous administration of 5-ASA is critical for prevention of acute UC flare ups and relapse. ${ }^{11}$ Therefore, treatment adherence is crucial for reducing the relapse rate. Previous studies have reported that it is easier to comply with and more convenient to take a once daily (OD) dose of 5-ASA than 2 to 3 daily doses. It was also reported that the effect of the drug was not affected by the type of dosing regimen. ${ }^{12-16}$ However, there have been no well-designed studies addressing this in Korea.

Thus, the aims of this clinical trial were to evaluate the adherence to different 5-ASA administration regimens and assess the latter's consequence on treatment efficacy.

\section{METHODS}

\section{Trial Design}

The study was a prospective, multicenter, randomized trial comparing adherence to an OD dose of $2.4 \mathrm{~g}$ mesalazine $\left(\right.$ Asacol $\left.^{\circledR}\right)$, with that to a divided daily (DD) regimen of mesalazine, either $800 \mathrm{mg} 3$ times daily or 1,200 mg twice daily, for maintenance therapy in UC during 12-month follow-up.

\section{Participants}

Patients with UC that extended beyond the sigmoid colon and who had been maintaining their remission (partial Mayo score of 2 or below) by taking 5-ASA compounds for at least 3 months were enrolled. Patients had to be older than 18 years, and able to give informed consent. Patients were excluded if they had a history of using biologic agent; had started or altered their dose of steroids, azathioprine/6-mercaptopurine or enema/suppository therapy for UC in the past 3 months (these drugs were permitted if a stable dosage was used over that period of time); had a history of colectomy; were breast feeding, pregnant, or there was a chance of being pregnant; had unstable psychiatric disease; were allergic to 5-ASA; showed no remission with 5-ASA treatment for 3 months; had severe or fulminant UC; had used more than $2.4 \mathrm{~g} /$ day of oral mesalazine containing products in the past 3 months; had heart failure, heart disease and other severe diseases; had other inflammatory disease and/or infectious colitis; had problems with liver or kidney function.

\section{Initial Evaluation, Randomization and Follow-up Evaluations}

At the first visit, enrolled patients provided written informed consent, and a sigmoidoscopy was performed. Mucosal inflammation was graded using the Mayo score with proctosigmoidoscopy (grade 0 , normal or inactive disease; grade 1, mild disease, erythema, decreased vascular pattern, mild friability; grade 2, moderate disease, marked erythema, absent vascular pattern, friability, erosions; grade 3, severe disease, spontaneous bleeding, ulceration). Symptoms of disease were also investigated by the Mayo score; the Full score incorporated the modified sigmoidoscopy score. Details of the patient's UC, other medical history, concomitant medication, and laboratory markers including complete blood count, ESR, CRP, creatinine (Cr), AST, ALT, and ALP, were obtained.

Patients were randomized to OD or DD treatment regimens for 1 year in a 1:1 ratio via computerized randomization. The patient's registration center allocated the study drugs randomly to the eligible subjects. The OD group had to take $2.4 \mathrm{~g}$ once daily and the DD group had to take $0.8 \mathrm{~g} 3$ times or $1.2 \mathrm{~g}$ twice a day. In the DD group, whether to take Asacol ${ }^{\circledR} 3$ times or 4 times was decided via the computerized randomization.

Routine follow-up was conducted every 3 months for a year (4 times in total). At every follow-up, the evaluators recorded adherence and the partial Mayo score, and also measured complete blood count, ESR, CRP, Cr, AST, ALT, and ALP. Patients had sigmoidoscopies at the time of randomization and at the end of the 12 months in order to assign a full Mayo score. Fecal calprotectin was measured at the 6-month and 12-month follow-ups. Samples were sent to an external laboratory (Green Cross laboratories, Yongin, Korea); clinicians did not know the calprotectin results during the study. To detect mild inflammation sensitively, the cutoff value of fecal calprotectin was set at $100 \mu \mathrm{g} / \mathrm{mL} .^{17-22}$ Patients were also asked how satisfied they were with their allocated regimen.

\section{Outcome}

The primary endpoint was the adherence rate to mesalazine $\left(\right.$ Asacol $^{\circledR}$ ) $2.4 \mathrm{~g}$ once daily, $800 \mathrm{mg} 3$ times daily and 1,200 mg twice daily, for maintenance treatment in UC during 12- month of follow-up. To evaluate adherence, the enrolled patients kept daily drug diaries (self-reported adherence) and research nurses (Yeiwon Choi and Yunmi Jung) counted their remaining drug at 3, 6, 9, and 12 months to confirm their compliance (tablet counts) ${ }^{23}$ Drug diaries was paper form and had tables in which patients could fill up the blanks whether they took a medicine as their regimen. Research nurse educated and checked patients' diaries every 3 months for 1 year. The adherence rate was calculated by dividing the number of tablets really taken 
by the number of tablets which should be taken.

The secondary endpoints were the relapse rate, treatment satisfaction, adverse events rate and laboratory markers change during the follow-up period. Relapse was defined as a partial Mayo score over 3 or any case where another modality, including another drug, an operation or hospital admission for treatment, was required to manage the UC. Satisfaction was classified into 1 of 4 categories (very satisfied, satisfied, dissatisfied, and very dissatisfied). The adverse events reported included nausea, vomiting, abdominal pain/cramp, headache, pyrexia, proctalgia, arthralgia, and others. The severity of the adverse events was categorized as mild, moderate, or severe. Mild meant that the event did not interfere with usual activity. Moderate meant that the event interfered with usual activity but the patient did not need to change the drug regimen. Severe meant that the patient needed to change the drug regimen due to the adverse event.

\section{Statistical Methods}

The sample size was calculated based on previous studies which reported that the adherence rate was $97 \%$ in the OD group and $85 \%$ in the DD group. ${ }^{24}$ A total of 192 patients (96 patients for each group) were needed to demonstrate superiority of the OD group (power $80 \%$, level of significance $5 \%$, and dropout rate $10 \%$ ). The modified intention-to-treat (ITT) population was defined as all randomized patients who had reported at least 1 adherence. To fill in missing values, the last observation carried forward imputation method was applied. Per-protocol (PP) population was defined as the patients who had reported adherence at all point (3, 6, 9, and 12 months).

All data were analyzed using SPSS version 24.0 (IBM Corp.,
Armonk, NY, USA). The chi-square test or Fisher exact test was used for the analysis of categorical variables (sex, smoking, history of relapse, disease location, co-medication, relapse rate, satisfaction, and laboratory marker change). Continuous variables (adherence rate, age, BMI, and UC duration) were analyzed using Student $t$-test or the Mann-Whitney $U$-test. Results were considered statistically significant when the $P$-value was $<0.05$. In the analysis of adherence at 4 point $(3,6,9$, and 12 months), the Bonferroni correction was applied in the multiple testing (statistically significant when the $P$-value was $<0.0125)$.

\section{Ethical Statement}

This clinical trial followed the principle of Good Clinical Practice and the Declaration of Helsinki. Trial protocol was reviewed and approved by the Kangbuk Samsung Hospital IRB (KBSMC201401084002). All subjects provided written informed consent

\section{RESULTS}

\section{Patient Population}

One hundred and eighty-two patients were enrolled between January 2015 and June 2016 (Fig. 1). Before randomization, 2 patients were excluded due to condition aggravation and withdrawal of consent. The remaining 180 patients were randomized (91 to the OD group, and 89 to the DD group). In the OD group, 13 patients were excluded (11 due to withdrawal of consent, and 2 due to follow-up loss). In the DD group, 8 patients were excluded ( 5 due to withdrawal of consent, 2 due to follow-up loss, and 1 due to pregnancy). Ultimately, data of the

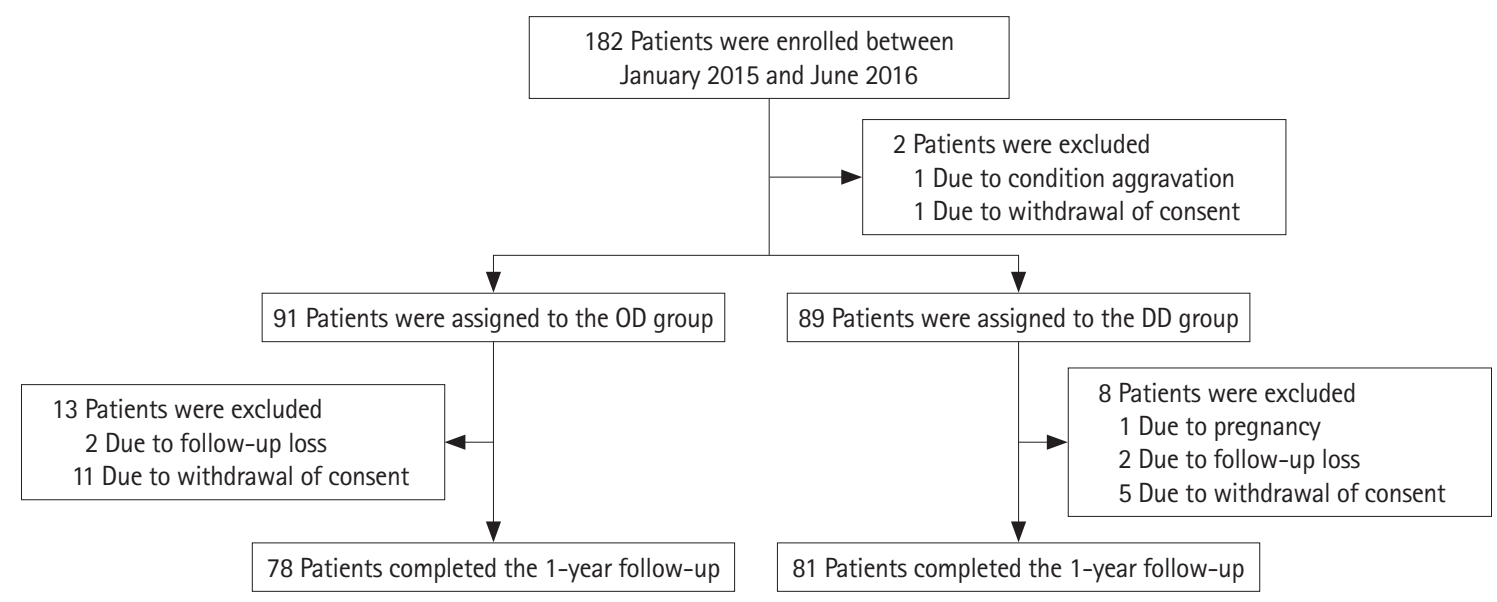

Fig. 1. Patient enrollment and follow-up during the trial. $O D$, once daily; $D D$, divided daily. 
180 patients who were randomized to each groups were analyzed (91 patients in the OD group and 89 patients in the DD group). In the DD group, 69 patients $(77.5 \%)$ received twice daily dosing of mesalazine and 20 patients $(22.5 \%)$ received

Table 1. Demographic and Clinical Details of the Patients

\begin{tabular}{lcccc}
\hline Characteristic & $\begin{array}{c}\text { Total } \\
(\mathrm{n}=180)\end{array}$ & $\begin{array}{c}\text { OD group } \\
(\mathrm{n}=91)\end{array}$ & $\begin{array}{c}\text { DD group } \\
(\mathrm{n}=89)\end{array}$ & P-value \\
\hline Age (yr) & $46.8 \pm 14.8$ & $45.7 \pm 13.8$ & $47.9 \pm 15.6$ & 0.30 \\
Male sex & $99(55.0)$ & $56(61.5)$ & $43(48.3)$ & 0.08 \\
BMI $\left(\mathrm{kg} / \mathrm{m}^{2}\right)$ & $22.8 \pm 2.9$ & $22.2 \pm 2.9$ & $23.3 \pm 2.8$ & 0.02 \\
Smoking & & & & 0.69 \\
Ex-smoker & $28(15.6)$ & $16(17.6)$ & $12(13.5)$ & \\
Current smoker & $20(11.1)$ & $9(9.9)$ & $11(12.4)$ & \\
UC duration (yr) & $7.1 \pm 8.2$ & $6.8 \pm 6.1$ & $7.5 \pm 9.9$ & 0.61 \\
History of relapse & $57(31.7)$ & $32(35.2)$ & $25(28.1)$ & 0.31 \\
Disease location & & & & 0.06 \\
$\quad$ Left sided & $115(63.9)$ & $52(57.1)$ & $63(70.8)$ & \\
Pancolitis & $65(36.1)$ & $39(42.9)$ & $26(29.2)$ & \\
Co-medication & & & & 0.54 \\
$\quad$ Suppositories & $50(27.8)$ & $24(26.4)$ & $26(29.2)$ & \\
Steroid & $8(4.4)$ & $4(4.4)$ & $4(4.5)$ & \\
Immunomodulator & $28(15.6)$ & $13(14.3)$ & $15(16.9)$ & \\
\hline
\end{tabular}

Values are presented as mean \pm SD or number (\%).

$\mathrm{OD}$, once daily; $\mathrm{DD}$, divided daily. thrice daily dose of mesalazine.

Baseline characteristics are shown in Table 1. The 2 groups were well-matched except for BMI. The average age was 46.8 years. Male patients comprised $55.0 \%$ of patients while female patients accounted for $45.0 \%$ of patients. The average UC duration was 7.1 years; $63.9 \%$ had left-sided colitis and $36.1 \%$ had pancolitis. History of relapse was reported in $31.7 \%$ of patients, whereas $68.3 \%$ never had relapse. BMI was the only characteristic that was significantly different between the 2 groups (22.2 in the OD group vs. 23.3 in the DD group, $P=0.02$ ).

\section{Primary Outcome}

All self-reported adherence rates and adherence rates measured by table count are shown in Table 2. In ITT population, both self-reported adherence (96.5\% vs. 94.3\%, $P=0.09$ ) and adherence measured by tablet counts ( $96.5 \%$ vs. $93.9 \%, P=0.04$ ) tended to show higher in the OD group than in the DD group at 6 months. However, when we apply Bonferroni correction $(P<0.0125)$, there was no statistical significance in both selfreported adherence and adherence measured by tablet counts at 6 months. In addition, at 1 year, both the self-reported adherence rate ( $95.6 \%$ vs. $96.0 \%, P=0.78)$ and the adherence rate measured by tablet counts ( $94.9 \%$ vs. $95.1 \%, P=0.89$ ) were not different between the 2 groups.

The proportion of patients whose adherence rate was lower than $90 \%$ is depicted in Fig. 2. At 6 months, the proportion of

Table 2. Self-Reported Adherence Rate and Adherence Rate Measured by Tablet Counts

\begin{tabular}{|c|c|c|c|c|c|c|}
\hline \multirow{2}{*}{ Adherence } & \multicolumn{2}{|c|}{ OD group } & \multicolumn{2}{|c|}{ DD group } & \multirow{2}{*}{$P$-value ${ }^{c}$} & \multirow{2}{*}{$P$-value } \\
\hline & $\mathrm{ITT}(\mathrm{n}=91)^{\mathrm{a}}$ & $\operatorname{PP}(n=78)^{b}$ & $\mathrm{ITT}(\mathrm{n}=89)^{\mathrm{a}}$ & $\mathrm{PP}(\mathrm{n}=81)^{b}$ & & \\
\hline \multicolumn{7}{|c|}{ Self-reported adherence $(\%)$} \\
\hline 3 Months & 96.6 & 97.1 & 96.3 & 96.6 & 0.77 & 0.57 \\
\hline 6 Months & 96.5 & 97.0 & 94.3 & 94.4 & 0.09 & 0.03 \\
\hline 9 Months & 96.4 & 97.1 & 95.6 & 95.7 & 0.54 & 0.23 \\
\hline 12 Months & 95.6 & 96.0 & 96.0 & 96.1 & 0.78 & 0.97 \\
\hline \multicolumn{7}{|c|}{ Adherence by tablet counts $(\%)$} \\
\hline 3 Months & 96.5 & 96.8 & 95.8 & 96.9 & 0.56 & 0.48 \\
\hline 6 Months & 96.5 & 96.9 & 93.9 & 94.0 & 0.04 & 0.02 \\
\hline 9 Months & 95.8 & 96.4 & 94.8 & 94.9 & 0.40 & 0.20 \\
\hline 12 Months & 94.9 & 95.2 & 95.1 & 95.1 & 0.89 & 0.92 \\
\hline
\end{tabular}

Significant at $P$-value $<0.0125$.

aIT means the modified intention-to-treat population.

${ }^{b} \mathrm{PP}$ means the per-protocol population.

${ }^{c} P$-value is for the modified intention-to-treat population.

${ }^{\mathrm{d}} P$-value is for the per-protocol population.

$\mathrm{OD}$, once daily; $\mathrm{DD}$, divided daily. 

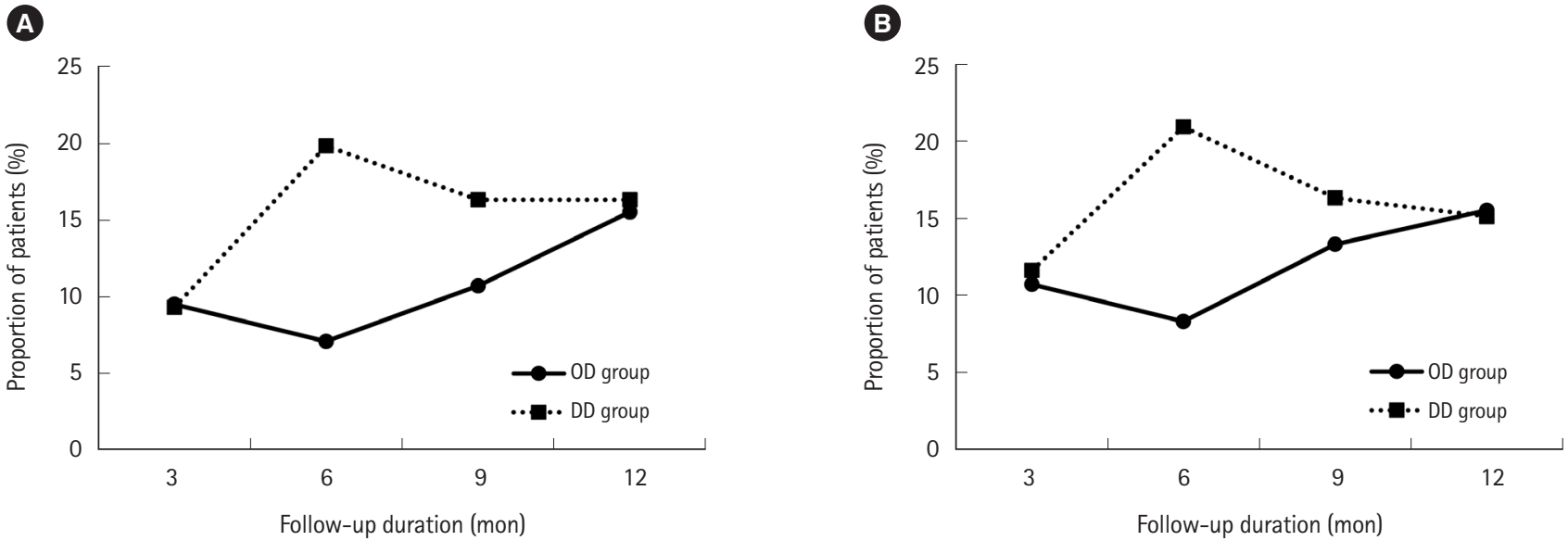

Fig. 2. Proportion of patients whose adherence was lower than 90\%. (A) Self-reported adherence. (B) Adherence measured by tablet counts in the once daily (OD) group versus the divided daily (DD) group.

Table 3. Comparison of Relapse Rate, Satisfaction and Laboratory Marker Change

\begin{tabular}{|c|c|c|c|}
\hline Secondary outcome & $\begin{array}{l}\text { OD group } \\
(n=91)\end{array}$ & $\begin{array}{l}\text { DD group } \\
(n=89)\end{array}$ & $P$-value \\
\hline Relapse & $8(8.8)$ & $4(4.5)$ & 0.37 \\
\hline$<6$ Months & $2(2.2)$ & 0 & 0.50 \\
\hline 6-12 Months & $6(6.6)$ & $4(4.5)$ & 0.75 \\
\hline Median duration (mon) & $6.8 \pm 6.1$ & $7.5 \pm 9.9$ & 0.93 \\
\hline Satisfaction & & & $<0.001$ \\
\hline Very satisfied & $64(78.0)$ & $40(48.8)$ & \\
\hline Satisfied & $16(19.5)$ & $40(48.8)$ & \\
\hline Dissatisfied & $2(2.4)$ & $2(2.4)$ & \\
\hline Very dissatisfied & 0 & 0 & \\
\hline \multicolumn{4}{|l|}{$\mathrm{ESR}>30 \mathrm{~mm} / \mathrm{hr}$} \\
\hline 6 Months & $8 / 72(11.1)$ & $3 / 74(4.1)$ & 0.13 \\
\hline 12 Months & $5 / 47(10.6)$ & $10 / 48(20.8)$ & 0.17 \\
\hline \multicolumn{4}{|l|}{$\mathrm{CRP}>0.6 \mathrm{mg} / \mathrm{dL}$} \\
\hline 6 Months & $9 / 73(12.3)$ & $15 / 76(19.7)$ & 0.22 \\
\hline 12 Months & $6 / 48(12.5)$ & $10 / 49(20.4)$ & 0.29 \\
\hline \multicolumn{4}{|l|}{ Calprotectin $>100 \mu \mathrm{g} / \mathrm{mg}$} \\
\hline 6 Months & 28/77 (36.4) & $33 / 78$ (42.3) & 0.45 \\
\hline 12 Months & $25 / 50(50.0)$ & 25/61 (41.0) & 0.34 \\
\hline
\end{tabular}

Values are presented as number (\%), means $\pm \mathrm{SD}$, or number/total $(\%)$. $\mathrm{OD}$, once daily; $\mathrm{DD}$, divided daily.

patients whose adherence rate was lower than $90 \%$ tended to lower in the OD group than in the DD group by self-reported adherence rate $(7.1 \%$ vs. $19.8 \%, P=0.02)$ and by adherence rate measured by tablet counts ( $8.3 \%$ vs. $20.9 \%, P=0.02)$. However when we apply Bonferroni correction, there was no sta-
Table 4. Comparison of Adverse Event Rate

\begin{tabular}{lccc}
\hline Adverse event & $\begin{array}{c}\text { OD group } \\
(\mathrm{n}=91)\end{array}$ & $\begin{array}{c}\text { DD group } \\
(\mathrm{n}=89)\end{array}$ & P-value \\
\hline Any AE & $12(13.2)$ & $13(14.6)$ & 0.78 \\
Nausea & $2(2.2)$ & $1(1.1)$ & 1.00 \\
Vomiting & 0 & $1(1.1)$ & 0.49 \\
Abdominal pain & $6(6.6)$ & $3(3.4)$ & 0.50 \\
Bloating & $5(5.5)$ & $7(7.9)$ & 0.52 \\
Headache & $2(2.2)$ & 0 & 0.50 \\
Pyrexia & 0 & 0 & - \\
Proctalgia & $1(1.1)$ & 0 & 1.00 \\
Arthralgia & $1(1.1)$ & $4(4.5)$ & 0.21 \\
Others & $2(2.2)$ & 0 & 0.50 \\
Severity of AE & & & 0.78 \\
Any mild AE & $12(13.2)$ & $13(14.6)$ & \\
Any moderate AE & 0 & 0 & \\
Any severe AE & 0 & 0 & \\
\hline
\end{tabular}

Values are presented as number (\%).

$O D$, once daily; $D D$, divided daily; $A E$, adverse event.

tistical significance. In addition, at 1 year, there was no difference in the proportion of patients whose adherence rate was lower than $90 \%$ in self-reported adherence rate (15.5\% vs. $16.3 \%$, $P=0.88)$ or in adherence rate measured by tablet counts (15.5\% vs. $15.1 \%, P=0.94$ ) between the OD group and the DD group respectively.

\section{Secondary Outcome}

Relapse rate, adverse event rate, satisfaction, and laboratory data were compared between the 2 groups (Tables 3 and 4). 
There was no significant difference in relapse rate between the 2 groups throughout the entire follow-up period. There were 8 cases (8.8\%) of relapse in the OD group, 4 cases (4.5\%) in the DD group $(P=0.37)$, during follow-up period. There was no significant difference in the median duration of relapse between the 2 groups ( 6.8 months vs. 7.5 months, $P=0.93$ ).

There were no statistically significant differences in adverse event rates between the 2 groups. In the OD group, 12 patients (13.2\%) reported adverse events; 2 had nausea (2.2\%), 6 had abdominal pain (6.6\%), 5 had bloating (5.5\%), 2 had headaches (2.2\%), 1 had proctalgia (1.1\%), 1 had arthralgia (1.3\%) and 2 had hematochezia which was classified as etc $(2.2 \%)$. In the DD group, 13 patients (14.6\%) reported adverse events; 1 had nausea (1.1\%), 1 had vomiting (1.1\%), 3 had abdominal pain (3.4\%), 7 had bloating (7.9\%) and 4 had arthralgia (4.5\%). The severity of the adverse events was also compared. There was no statistically significant difference in the severity of adverse events between the 2 groups $(P=0.78)$. All adverse events were reported as mild.

For patient satisfaction, statistically significant differences were observed between the 2 groups. Patients in the OD group were more satisfied with their drug administration regimen than patients in the DD group $(P<0.001)$. In the OD group, 64 patients $(78.0 \%)$ were very satisfied with their treatment regimen, whereas only 40 patients $(48.8 \%)$ were very satisfied in the DD group. In the OD group, 16 patients (19.5\%) were satisfied with their treatment, whereas 40 patients (48.8\%) were satisfied in the DD group.

Serum ESR, CRP, and fecal calprotectin were analyzed at 6 months and 1 year. There was no statistically significant difference among the median ESR, CRP, and fecal calprotectin levels of the 2 groups.

\section{DISCUSSION}

Both self-reported adherence rate and adherence rate measured by tablet counts were not different between OD and DD group. In addition there was no difference in the relapse rate between the 2 groups. However, patients in the OD group were more satisfied with their drug administration regimen than patients in the DD group.

5-ASA is currently established as a standard treatment for first-line treatment of mild-to-moderate UC. 5-ASA proved effective and is tolerable by the patients for the treatment of active disease and the maintenance of remission. Both oral and topical 5-ASA formulations are inconvenient; in particular, the oral formulations require multiple daily dosing with multiple tablets per dose. Such demanding regimens could be hard on the everyday lives of patients and aggravate their overall quality of life. The problem with complex and uncomfortable dosing regimen is that they can lower the adherence, which can lead to poor prognosis. Previous studies have reported that the adherence to oral 5-ASAs for the patients with quiescent UC was low enough to take only $40 \%$ of the regimen. ${ }^{25,26}$ Part of this could be explained by the uncomfortable dosing regimens, particularly if taken 3 times daily. ${ }^{11}$ OD, unlike DD regimen, is preferred for patients because it is easy to administrate. This preference may lead to improved adherence to therapy.

The randomized control trials conducted with various preparations of mesalazine have demonstrated that OD regimens are not inferior than DD regimens for both induction and maintenance of remission. ${ }^{12-16,24,27,28}$ However, compliance was not significantly different between the 2 groups. In the QDIEM study, adherence was measured using the Medication Adherence Report Scale medication adherence questionnaire, but without tablet counts, and the only significant difference in reported adherence was at 3 months (in favor of OD treatment); nevertheless adherence remained very high throughout the study. ${ }^{13}$ Another maintenance trial with mesalazine tablets also showed high adherence in both the once- and twice-daily arms. ${ }^{16}$ In the PODIUM study the visual analog score for adherence was significantly better for the OD group at visits 2 and 3 (compared to twice daily) but not at the end of the trial, and sachet counts did not uncover a difference in adherence. ${ }^{12}$ However, as the primary endpoint of these studies was recurrence rate, they did not have enough power to detect small statistically significant differences in compliance. Thus, we aim to investigate adherence to mesalazine $\left(\right.$ Asacol $\left.^{\circledR}\right)$ by comparing an OD and DD regimen for maintenance therapy in UC during 12-month of follow-up.

Although we expected improved adherence when taking medicines once-daily compared with twice or 3 times daily, this was not confirmed in our study. Although adherence tended to show higher in the OD group than in the DD group at 6 months, there was no statistical significance after applying Bonferroni correction. This result corresponds with that of previous studies that have shown a significant difference in reported adherence only at 3 months and 4 months. ${ }^{12,13}$ We observed excellent rates of compliance with Asacol ${ }^{\circledR}$ in $95 \%$ of patients or more, and the reason might be that in a clinical trial setting, participants are generally more motivated and closely monitored by the medical staff. As a result, the relapse rate 
was low in both the OD and DD groups. However, the patients' satisfaction was higher in the OD than in the DD group, as we expected from previous studies. ${ }^{13,14,24}$

The strength of our study is that it could detect statistically significant difference in compliance between the OD and DD group; we chose adherence as the primary endpoint, not relapse rate. However, our study has limitations. First, such as the subjectivity associated with patient reported adherence. Paper form drug diaries and checking diaries every 3 months by research nurse might increase compliance, and more objective method should have been used such as the cap opening method. ${ }^{24}$ Cap opening data provide objective evidence, although the assumption is made that patients actually ingested tablets in the correct dose after opening the bottle. A previous study that investigated adherence with the cap opening method reported significantly better adherence in the OD group. ${ }^{24}$ In addition, it is well recognized that adherence is significantly worse for any 3 times daily drug regimen, compared to twice daily, whereas the adherence difference is less dramatic between twice-daily and OD treatment. ${ }^{29}$ In our study, the proportion of patients on twice-daily dosing (76.5\%) was higher than that on thrice-daily dosing (23.5\%). Second, as the primary endpoint of this study was the adherence rate to 5-ASA, not the recurrence, we enrolled patients who were using concomitant immunomodulator. Concomitant immunomodulator might affect low recurrence rate. However, recurrence rate was not different between 2 groups after we excluded these patients (OD group 7.7\% vs. DD group 5.4\%, $P=0.75$ ).

In conclusion, although adherence was not different between the OD group and DD group, the patients' satisfaction was higher in the OD than in the DD group. The relapse rate between the 2 groups was not significantly different, indicating that OD dosing might have the same effect as DD for the maintenance of UC remission.

\section{FINANCIAL SUPPORT}

The authors received no financial support for the research, authorship, and/or publication of this article.

\section{CONFLICT OF INTEREST}

No potential conflict of interest relevant to this article was reported.

\section{AUTHOR CONTRIBUTION}

Conceptualization: Park DI. Methodology: Park DI, Park SK. Investigation: Park DI, Park SK, Eun CS, Seo GS, Im JP, Kim TO. Formal analysis: Park SK, Park SH. Project administration: Park DI. Visualization: Park SK, Park SH. Writing-original draft: Park SH. Writing-review and editing: Park DI, Park SK. Approval of final manuscript: all authors.

\section{REFERENCES}

1. Choi CH, Kim YH, Kim YS, et al. Guidelines for the management of ulcerative colitis. Korean J Gastroenterol 2012;59:118140.

2. Choi CH, Moon W, Kim YS, et al. Second Korean guidelines for the management of ulcerative colitis. Intest Res 2017;15:737.

3. Wei SC, Chang TA, Chao TH, et al. Management of ulcerative colitis in Taiwan: consensus guideline of the Taiwan Society of Inflammatory Bowel Disease. Intest Res 2017;15:266-284.

4. Hanauer SB, Sninsky CA, Robinson M, et al. An oral preparation of mesalamine as long-term maintenance therapy for ulcerative colitis: a randomized, placebo-controlled trial. Ann Intern Med 1996;124:204-211.

5. Kao J, Kwok K, Das KM. Inducing and maintaining remission in ulcerative colitis: role of high-dose, extended-release mesalamine. J Clin Gastroenterol 2010;44:531-535.

6. Wang Y, Parker CE, Bhanji T, Feagan BG, MacDonald JK. Oral 5-aminosalicylic acid for induction of remission in ulcerative colitis. Cochrane Database Syst Rev 2016;4:CD000543.

7. Dew MJ, Hughes P, Harries AD, Williams G, Evans BK, Rhodes J. Maintenance of remission in ulcerative colitis with oral preparation of 5-aminosalicylic acid. Br Med J (Clin Res Ed) 1982; 285:1012.

8. Hibi T, Ueno F, Matsuoka K, Lee T. Guidelines for the management of ulcerative colitis in Japan: developed through integration of evidence and consensus among experts. IBD Res 2010;4:189-239.

9. Mayberry J. The history of 5-ASA compounds and their use in ulcerative colitis: trailblazing discoveries in gastroenterology. J Gastrointestin Liver Dis 2013;22:375-377.

10. Iacucci M, de Silva S, Ghosh S. Mesalazine in inflammatory bowel disease: a trendy topic once again? Can J Gastroenterol 2010;24:127-133.

11. Kane SV. Systematic review: adherence issues in the treatment of ulcerative colitis. Aliment Pharmacol Ther 2006;23:577-585. 
12. Dignass AU, Bokemeyer B, Adamek H, et al. Mesalamine once daily is more effective than twice daily in patients with quiescent ulcerative colitis. Clin Gastroenterol Hepatol 2009;7:762769.

13. Sandborn WJ, Korzenik J, Lashner B, et al. Once-daily dosing of delayed-release oral mesalamine (400-mg tablet) is as effective as twice-daily dosing for maintenance of remission of ulcerative colitis. Gastroenterology 2010;138:1286-1296.

14. Kruis W, Kiudelis G, Rácz I, et al. Once daily versus three times daily mesalazine granules in active ulcerative colitis: a doubleblind, double-dummy, randomised, non-inferiority trial. Gut 2009;58:233-240.

15. Suzuki Y, Iida M, Ito H, et al. $2.4 \mathrm{~g}$ Mesalamine (Asacol $400 \mathrm{mg}$ tablet) once daily is as effective as three times daily in maintenance of remission in ulcerative colitis: a randomized, noninferiority, multi-center trial. Inflamm Bowel Dis 2017;23:822832.

16. Kamm MA, Lichtenstein GR, Sandborn WJ, et al. Randomised trial of once- or twice-daily MMX mesalazine for maintenance of remission in ulcerative colitis. Gut 2008;57:893-902.

17. Scaioli E, Scagliarini M, Cardamone C, et al. Clinical application of faecal calprotectin in ulcerative colitis patients. Eur J Gastroenterol Hepatol 2015;27:1418-1424.

18. Scaioli E, Digby RJ, Belluzzi A. Different cutoff levels of fecal calprotectin to predict clinical relapse in ulcerative colitis. Inflamm Bowel Dis 2016;22:E26.

19. Chang S, Malter L, Hudesman D. Disease monitoring in inflammatory bowel disease. World J Gastroenterol 2015;21:1124611259.

20. Theede K, Holck S, Ibsen P, Kallemose T, Nordgaard-Lassen I, Nielsen AM. Fecal calprotectin predicts relapse and histological mucosal healing in ulcerative colitis. Inflamm Bowel Dis 2016;22:1042-1048.
21. García-Sánchez V, Iglesias-Flores E, González R, et al. Does fecal calprotectin predict relapse in patients with Crohn's disease and ulcerative colitis? J Crohns Colitis 2010;4:144-152.

22. Yamamoto T, Shiraki M, Bamba T, Umegae S, Matsumoto K. Fecal calprotectin and lactoferrin as predictors of relapse in patients with quiescent ulcerative colitis during maintenance therapy. Int J Colorectal Dis 2014;29:485-491.

23. Chan W, Chen A, Tiao D, Selinger C, Leong R. Medication adherence in inflammatory bowel disease. Intest Res 2017;15: 434-445.

24. Hawthorne AB, Stenson R, Gillespie D, et al. One-year investigator-blind randomized multicenter trial comparing Asacol $2.4 \mathrm{~g}$ once daily with $800 \mathrm{mg}$ three times daily for maintenance of remission in ulcerative colitis. Inflamm Bowel Dis 2012;18: 1885-1893.

25. Kane SV, Cohen RD, Aikens JE, Hanauer SB. Prevalence of nonadherence with maintenance mesalamine in quiescent ulcerative colitis. Am J Gastroenterol 2001;96:2929-2933.

26. Shale MJ, Riley SA. Studies of compliance with delayed-release mesalazine therapy in patients with inflammatory bowel disease. Aliment Pharmacol Ther 2003;18:191-198.

27. Kruis W, Jonaitis L, Pokrotnieks J, et al. Randomised clinical trial: a comparative dose-finding study of three arms of dual release mesalazine for maintaining remission in ulcerative colitis. Aliment Pharmacol Ther 2011;33:313-322.

28. Prantera C, Kohn A, Campieri M, et al. Clinical trial: ulcerative colitis maintenance treatment with 5-ASA: a 1-year, randomized multicentre study comparing MMX with Asacol. Aliment Pharmacol Ther 2009;30:908-918.

29. Eisen SA, Miller DK, Woodward RS, Spitznagel E, Przybeck TR. The effect of prescribed daily dose frequency on patient medication compliance. Arch Intern Med 1990;150:1881-1884. 